\title{
Transversal investigation of the risk of valproic-acid-induced tremor in a secondary epilepsy center: clinical, neuroimaging, and genetic factors
}

\author{
Lili Lan ${ }^{1}$, Xu Zhao ${ }^{1}$, Si Jian ${ }^{1}$, Cun $^{1}{ }^{1}$, Man Wang ${ }^{1}$, Qing Zhou ${ }^{1}$, Shanshan Huang ${ }^{1}$, \\ Suiqiang Zhu ${ }^{1}$, Huicong Kang ${ }^{1}$, and Heidi Kirsch ${ }^{2}$ \\ ${ }^{1}$ Tongji Hospital of Tongji Medical College of Huazhong University of Science and \\ Technology \\ ${ }^{2} \mathrm{UCSF}$
}

July 28, 2020

\begin{abstract}
Aim: To determine the risk factors of valproic acid (VPA)-induced tremor, with particular attention on characterizing cerebellar atrophy and identifying tremor-susceptible gene mutations. Methods: Epileptic patients taking VPA were divided into two groups, a tremor and a non-tremor group, based on self-reported or clinically assessed tremors. A mutation of rs9652490 in the leucine-rich repeat and immunoglobulin domain-containing Nogo-receptor-interacting protein 1 (LINGO-1) gene was determined by Sanger sequencing. Cerebellar atrophy was assessed and various cerebellar dimensions were measured on magnetic resonance imaging (MRI) scans. Results: Among 200 subjects enrolled, 181 were included for analysis (mean age $33.28 \pm 11.78$ years old, male:female $=2.77: 1)$. In the tremor group, the percentage of females $(\mathrm{p}=0.036)$, positive tremor family history $(\mathrm{p}=0.001)$, and incidence of polytherapy $(\mathrm{p}=0.034)$, treatment duration $(>12$ months $[\mathrm{p}=0.013]$ or $>24$ months $[\mathrm{p}=0.008])$, and daily dosage $(>1,000 \mathrm{mg} / \mathrm{d} ; \mathrm{p}=0.003)$ of VPA, were significantly higher than in the non-tremor group. Treatment with VPA magnesium $(\mathrm{p}=0.030)$, alone or in combination with carbamazepine $(\mathrm{p}=0.040)$, reduced the incidence of tremor. Furthermore, 176 gene sequencing results ruled out any significant difference between the two groups in the mutation of rs9652490 within LINGO-1 ( $\mathrm{p}=0.443) ; 86$ subjects' MRI scans indicated no significant differences in the ratio of cerebellar atrophy or the cerebellar-dimension values ( $\mathrm{p}>0.05$ ). However, mutation of rs9652490 within LINGO-1 was correlated with increased cerebellar atrophy $(\mathrm{p}=0.001)$, reduced cerebellar-hemisphere thickness $(\mathrm{p}=0.025)$, and right-cerebellar-hemisphere longitudinal diameter $(\mathrm{p}=0.047)$. Conclusion: Our cohort indicated risk and protective factors of VPA-induced tremor. Although mutation of rs9652490 within LINGO-1 correlated with cerebellar atrophy, neither was correlated with VPA-induced tremors.
\end{abstract}

\begin{abstract}
Aim:To determine the risk factors of valproic acid (VPA)-induced tremor, with particular attention on characterizing cerebellar atrophy and identifying tremor-susceptible gene mutations.

Methods: Epileptic patients taking VPA were divided into two groups, a tremor and a non-tremor group, based on self-reported or clinically assessed tremors. A mutation of rs9652490 in the leucine-rich repeat and immunoglobulin domain-containing Nogo-receptor-interacting protein 1 (LINGO-1) gene was determined by Sanger sequencing. Cerebellar atrophy was assessed and various cerebellar dimensions were measured on magnetic resonance imaging (MRI) scans.
\end{abstract}

Results: Among 200 subjects enrolled, 181 were included for analysis (mean age 33.28 \pm 11.78 years old, male:female $=2.77: 1)$. In the tremor group, the percentage of females $(p=0.036)$, positive tremor family history $(p=0.001)$, and incidence of polytherapy $(p=0.034)$, treatment duration $(>12$ months $[p=0.013]$ 
or $>24$ months $[p=0.008])$, and daily dosage $(>1,000 \mathrm{mg} / \mathrm{d} ; p=0.003)$ of VPA, were significantly higher than in the non-tremor group. Treatment with VPA magnesium $(p=0.030)$, alone or in combination with carbamazepine $(p=0.040)$, reduced the incidence of tremor. Furthermore, 176 gene sequencing results ruled out any significant difference between the two groups in the mutation of rs9652490 within LINGO-1 ( $p$ $=0.443) ; 86$ subjects' MRI scans indicated no significant differences in the ratio of cerebellar atrophy or the cerebellar-dimension values $(p>0.05)$. However, mutation of rs9652490 within LINGO-1 was correlated with increased cerebellar atrophy $(p=0.001)$, reduced cerebellar-hemisphere thickness $(p=0.025)$, and rightcerebellar-hemisphere longitudinal diameter $(p=0.047)$.

Conclusion: Our cohort indicated risk and protective factors of VPA-induced tremor. Although mutation of rs9652490 within LINGO-1 correlated with cerebellar atrophy, neither was correlated with VPA-induced tremors.

Keywords: epilepsy; valproic acid; LINGO-1; cerebellar atrophy; essential tremor

\section{Introduction}

Fifty-two years after being licensed, valproic acid (VPA) has become one of the most extensively prescribed antiepileptic drugs (AEDs) worldwide[1] and is indicated for diverse types of seizures, migraines, and bipolar disorder. VPA is generally considered to be safe and well-tolerated, but still induces adverse side effects clinically[2]. One of the most common adverse effects is tremor, as reported in $6-45 \%$ of patients[3-7], which causes reduced tolerance, long-term retention, and reduced quality of life, especially in patients who primarily rely on dexterous hands for their work.

Clinical observations at early stages have focused on the risk factors of VPA-induced tremor and have attempted to identify predictors for avoiding VPA in high-risk patients, albeit with inconsistent conclusions across studies. One early study found that the incidence of tremor was associated with a higher daily dose $(>750 \mathrm{mg} / \mathrm{d})$ of VPA rather than its serum concentration[3], and another study reported that one patient with a family history of tremor presented with an earlier tremor onset[4]. A study from Egypt found that in patients who accepted non-extended-release VPA therapy, older age (37.86 \pm 2.44 years in the tremor group compared with $30.43 \pm 3.15$ years in the non-tremor group), longer treatment duration ( $15.98 \pm 2.75$ years in the tremor group compared with $8.03 \pm 3.43$ years in the non-tremor group) and higher serum concentration of VPA $(90.05 \pm 25.06 \mu \mathrm{g} / \mathrm{ml}$ in the tremor group compared with $70.35 \pm 25.08 \mu \mathrm{g} / \mathrm{ml}$ in the non-tremor group) were risk factors for tremor. Furthermore, lower concentrations of dopamine and norepinephrine were correlated with VPA-induced tremor[8]. Another single-center study indicated that a long duration of epilepsy and a high-daily VPA dosage were risk factors for tremor, whereas gender was not[9]. On the contrary, another study found that women suffered more from VPA-induced tremor[10].

Further clinical research has indicated that VPA-induced tremor is characterized by postural and kinetic tremors observed in the upper limbs, which resemble essential tremor (ET) in terms of their similar frequencies, amplitudes, and distributions[10, 11]. Additionally, a surface-electromyography study confirmed ET-like manifestations during VPA-induced tremor, in terms of similar frequencies, rhythmicities, mean amplitudes, burst patterns, and burst durations[6]. Meanwhile, another study has indicated that VPA-induced tremor may benefit from propranolol, a beta-blocker recommended for ET treatment, when a reduced dose of VPA is not appropriate[12]. Therefore, many studies have confirmed that VPA-induced tremor resembles ET $[3,4,6,7]$, suggesting that they may share similar pathogenic mechanisms.

Three putative mechanisms of ET include cortico-olivo-cerebello-thalamic circuit impairment, $\gamma$-amino butyric acid (GABA)-ergic dysfunction, and cerebellar degeneration[13]. Clinically, based on the high prevalence of kinetic tremor and ataxia in ET patients, many studies have focused on elucidating cerebellar dysfunction in ET[14, 15]. Functional magnetic resonance imaging (fMRI) and positron emission tomography (PET) have been used to confirm reduced cerebellar metabolism in ET patients[16, 17]. Postmortem studies of ET patients have also identified injury to cerebellar Purkinje cells, including torpedoes and fusiform axonal thickenings, as well as increased axonal collateralization, branching, and sprouting[18]; strikingly, as many as $31.3 \%$ of Purkinje cells have been found to be absent in some ET cases[19]. Additionally, genome-wide 
association studies (GWAS) have revealed correlations between ET susceptibility and genetic variations affecting cerebellar function, of which the single-nucleotide polymorphism (SNP) of rs9652490 within the gene encoding leucine-rich repeat and immunoglobulin domain-containing Nogo-receptor-interacting protein 1 (LINGO-1) has been demonstrated to be most important[20]. Additional studies and meta-analyses have confirmed the association of LINGO-1 gene variants with ET[21-24]. Identified mutations in LINGO-1 result in elevated protein expression of LINGO-1, which is a protein that inhibits neuronal differentiation and myelination, and likely decreases the survival of Purkinje cells[25]. Additionally, LINGO-1 expression has been shown to be significantly increased in the cerebellum of ET patients, as compared with that in patients with Parkinson's disease (PD) or in age-matched healthy controls[26]. Collectively, these findings indicate that mutations in LINGO-1 contribute to ET susceptibility through inducing cerebellar dysfunction via decreasing survival of Purkinje cells. Moreover, the function of LINGO-1 has been shown to be mainly driven by rs9652490 alleles, which regulate LINGO-1 expression profiles especially when combined with certain epigenetic factors, such as drugs[25-27].

Although the mechanisms of VPA-induced tremor remain unknown, its ET-like manifestations suggest that investigation of cerebellar-related factors may be useful for revealing possible mechanisms and related risk biomarkers. Therefore, our present study included the following aims: (1) to identify risk factors of VPAinduced tremor, including clinical factors, LINGO-1 gene variation, and cerebellar morphological changes in structural MRI scans; and (2) to determine whether the LINGO-1 gene is associated with cerebellar morphological changes in structural MRI scans.

\section{Materials and methods}

\subsection{Subjects}

We collected and studied a cohort of 200 consecutive epileptic patients attending the epilepsy clinic of Tongji Hospital, affiliated with the Tongji Medical College of Huazhong University of Science and Technology, from September 2018 to February 2019. All patients were diagnosed with epilepsy according to the definition of the International League Against Epilepsy (ILAE) criteria of 2014[28]. Inclusion criteria for patients consisted of the following: (1) 18 years or older; and (2) taking VPA at $500 \mathrm{mg}$ per day or more for at least one month without leakage. Furthermore, exclusion criteria for patients consisted of the following: (1) unable to complete assessments for significant neurological deficits including dystonia, cognitive impairment, and severe psychosis; (2) tremor emerging before VPA intake or independent of VPA; (3) presence of comorbid diseases causing tremor including PD, hyperthyroidism, and drug abuse; and (4) patients taking medications other than AEDs with known anti-tremor or tremor-inducing effects including beta blockers, antipsychotics, and bronchodilators[29].

All patients were divided into two groups, a tremor group and a non-tremor group, based on whether or not 1) they confirmed that they experienced upper-limb tremors when asked and 2) a clinical professional trained in evaluating movement disorders confirmed the presence of these tremors.

\subsection{Clinical data}

Demographic information including age, gender, and family history were documented according to the patients' statements and their medical records. Clinical data included seizure type and frequency, disease duration, physical examination, electroencephalogram (EEG) data, and structural MRI data.

Details of VPA treatment regarding formulation (including extended release [ER] or non-ER, as well as sodium or magnesium valproate), daily dosage, duration, combined AEDs, and related side effects were also documented. To determine the association of the daily dosage of VPA with the incidence of tremor, we assigned two cut-off values in terms of VPA dosage: $750 \mathrm{mg} / \mathrm{d}$ and 1,000 mg/d. The VPA medication duration was also divided by two cut-off values, 12 months and 24 months, to determine the correlation between VPA treatment duration and the incidence of tremor.

\subsection{Tremor evaluation scale}


Tremor was evaluated via the Essential Tremor Rating Assessment Scale (TETRAS), version 3.1. The TETRAS is a scale developed by the Tremor Research Group, aiming at conveniently and efficiently quantifying ET severity and its influence on activities of daily life. TETRAS consists of two subscales, the Activities of Daily Living (ADL) Subscale - which contains 12 self-scored items, with a rating of 0-4 for each tremor's impact on ADL - and the Performance Subscale, which is comprised of nine items rated from 0-4 (among which half-point increments are allowed for two items) and four items that are rated bilaterally for all limbs (for a total of 16 scores). The maximum scores are 48 for the ADL Subscale, and 64 for the Performance Subscale, yield a maximum total score of 112 for the TETRAS[30, 31].

\subsection{Sequence of SNP rs9652490 in the LINGO-1 gene}

The validated SNP rs9652490 in the LINGO-1 gene was sequenced to determine if it is a possible risk factor for VPA-induced tremor. Genomic DNA was extracted from peripheral blood leukocytes via spin columns (FastPure blood DNA isolation mini kit, Vazyme Biotech Co., Ltd.). Primer design and genotyping assays were performed by TsingKe Biological Technology (Wuhan, China). Primers for polymerase chain reaction were TAGGTGCAAGGCCAGGATTG (forward) and CCACCTCACTGCTGCATGG (reverse). Then, Sanger sequencing was conducted on samples that were blinded to the technicians.

\subsection{Structural MRI scanning protocol and morphological analysis of the cerebellum}

Subjects who underwent head MRI scans at Tongji Hospital at one month prior to enrollment were included in morphological evaluations and cerebellar analyses. All MRI sequences were performed on a 3.0-T MRI scanner (Discovery MR750, GE Healthcare, Milwaukee, Wisconsin) with a 32-channel phased-array head coil. All included subjects for these experiments underwent standard structural MRI scans that included the following: axial T1-fluid-attenuated inversion recovery (FLAIR) (repetition time/echo time/inversion time $(\mathrm{TR} / \mathrm{TE} / \mathrm{TI})$ at $2,991 / 24 / 868 \mathrm{~ms}$; matrix size of $320 \times 320$; field of view (FOV) of $24 \times 24 \mathrm{~cm}^{2}$, slice thickness of $5 \mathrm{~mm}$, gap of $1.5 \mathrm{~mm}$ ); T2-weighted fast spin echo (FSE) (TR/TE at 4,579/102 ms, matrix size of $320 \times 224$, FOV of $24 \times 24 \mathrm{~cm}^{2}$, echo train length of 20 , slice thickness of $5 \mathrm{~mm}$, gap of $1.5 \mathrm{~mm}$ ); and T2-FLAIR (TR/TE/TI at $8,000 / 160 / 2,100 \mathrm{~ms}$, matrix size of $256 \times 256$, FOV of $24 \times 24 \mathrm{~cm}^{2}$, slice thickness of $5 \mathrm{~mm}$, gap of $1.5 \mathrm{~mm}$ ).

A total of 86 images were evaluated, and the corresponding cerebellar dimensions were measured by two experienced radiologists. Dimensions of cerebellar-hemisphere thickness, cerebellar-vermis thickness, left and right cerebellar-hemisphere longitudinal diameters, fourth-ventricle height, left and right middle-cerebellarpeduncle widths, cerebellar diameter, and head diameter were measured and compared between the two groups. Detailed locations and measurements are presented in Figure 1. Additionally, general observations regarding the existence of cerebellar atrophy were recorded to compare the incidence of cerebellar atrophy between the two groups.

\subsection{Statistical analysis}

Statistical analyses were conducted using IBM SPSS Statistics Software (version 21.0, SPSS, Inc., an IBM company, Chicago, IL, USA). Continuous variables are reported as the mean \pm standard deviation. Categorical variables are presented by counts $(\%)$. Independent-sample t-tests were conducted to compared the means of continuous variables between the two groups. Chi-square tests were used to compare the proportions of counts between the two groups, and Fisher's exact tests were applied to determine differences in categorical variables with small sample sizes (total sample number $<40$ or any expected number $<5$ ). A p value $<0.05$ was considered to be statistically significant.

\section{Results}

3.1 Demographic characteristics and basic clinical data

Among the 200 enrolled patients, 181 patients were included in the final analysis (19 were excluded due to being unable to provide crucial clinical information for data analysis). Among the analyzed patients, 94 
subjects were included in the tremor group whereas 87 subjects were included in the non-tremor group. The mean age was $33.28 \pm 11.78$ years and the male:female ratio was 2.77:1 (Table 1 ).

In our cohort, the mean disease duration was $11.41 \pm 9.29$ years and the mean age of onset was $21.79 \pm 12.81$ years. For seizure types based on the ILAE 2017 classification system [32], 126 of 181 subjects (69.6\%) presented with focal onset seizures. The overall abnormal rate of MR and CT was $64.5 \%(81 / 125)$, while that of routine and long-term EEG was $61.5 \%(83 / 135)$. In our epileptic cohort taking VPA, 36 subjects $(19.9 \%)$ were in the monotherapy group and 145 subjects $(80.1 \%)$ were in the polytherapy group. The mean daily dosage of VPA was $889.78 \pm 223.59 \mathrm{mg}$, and the mean duration of VPA treatment was $29.66 \pm 35.83$ months (Table 1). For the tremor group, the TETRAS total score was $25.41 \pm 10.49$, the ADL Subscale score was $7.29 \pm 5.94$, and the Performance Subscale score was $18.13 \pm 5.87$ (Table 1).

\subsection{Clinical risk factors correlated with VPA-induced tremor}

By comparing the clinical features of the two groups in our cohort, several clinical factors associated with VPA-induced tremor were revealed. Women suffered from VPA-related tremor more than men, as revealed by an incidence of $64.6 \%(31 / 48)$ in women compared to $47.4 \%(63 / 133)$ in men $(p=0.041)$. The percent of subjects with a family history of tremor (PD excluded) was higher in the tremor group, which was $20.2 \%$ $(19 / 94)$ compared to that of only $3.5 \%(3 / 87)$ in the non-tremor group $(p=0.001)$ (Table 1$)$. A daily dosage of VPA that was higher than 1,000 mg was associated with an increased incidence of tremor $(12.8 \%, 12 / 94)$ compared to that in the non-tremor group $(1.2 \%, 1 / 87 ; p=0.003)$ (Table 2$)$. There was a significant difference in the VPA therapy duration between the tremor group (35.08 \pm 39.96 months) and the non-tremor group (21.62 \pm 25.89 months; $p=0.007)$. An increased risk of tremor was found when the VPA therapy duration was longer than 12 months ( $p=0.032)$ or 24 months $(p=0.008)$, as compared with those less than 12 or 24 months (Table 2). Taking VPA sodium-extended-release (ER) was also a risk factor of tremor compared to that of taking VPA magnesium-ER $(p=0.030)$, whereas there was no significant difference in the risk of tremor between patients taking ordinary VPA versus those taking VPA-ER $(p=0.233)$ (Table 2$)$. Additionally, there were more patients under polytherapy in the tremor group compared with those in the non-tremor group $(p$ $=0.034)$. Furthermore, a combination of VPA $+\mathrm{CBZ}$ was found to decrease the incidence of tremor compared with that of VPA+any other AEDs $(p=0.040)$; however, no statistical differences were found when VPA was combined with topiramate (TPM), lamotrigine (LTG), oxcarbazepine (OXC), or levetiracetam (LEV) $(p>0.05)$ (Table 3). Finally, age $(p=0.103)$, disease duration $(p=0.233)$, age at onset $(p=0.526)$, seizure type $(p=0.072)$, neuroimaging abnormalities $(p=0.280)$, and EEG abnormalities $(p=0.713)$ had no effects on VPA-induced tremor (Table 1).

3.3 VPA-induced tremor is not correlated with cerebellar atrophy or morphological changes in structural MRI scans

Among 86 structural MR images scanned at our hospital for analysis, 29 images (33.7\%) were considered to exhibit cerebellar atrophy. The number of patients presenting with cerebellar atrophy in structural MRI scans was not significantly different between the tremor group $(51.7 \%, 15 / 29)$ and non-tremor group $(50.0 \%$, $14 / 28)(p=0.941)$ (Table 6). Meanwhile, there was also no difference in each of the cerebellar dimensions measured on structural MR images between the tremor and non-tremor group $(p>0.05)$ (Table 4$)$.

\subsection{Mutation of rs9652490 in LINGO-1 does not increase the risk of VPA-induced tremor}

A total of 176 subjects out of the 181 total cohort accepted and underwent Sanger sequencing, among whom the SNP rs9652490 within LINGO-1 was detected in 63 subjects. We found that $38.5 \%$ of subjects (35/91) in the tremor group versus $32.9 \%$ in the non-tremor group (28/85) were carrying the SNP rs9652490 within LINGO-1, revealing that there was no statistical difference in this parameter between the two groups $(p$ $=0.443)($ Table 5$)$.

3.5 Mutation of rs9652490 in LINGO-1 is associated with cerebellar atrophy and a reduction in specific cerebellar dimensions in structural MRI scans

Carrying the SNP rs9652490 within LINGO-1 significantly increased the incidence of cerebellar atrophy 
when compared with those not carrying this SNP, with $62.1 \%(18 / 29)$ versus $37.9 \%(11 / 29)$ of patients exhibiting cerebellar atrophy with and without SNP rs9652490, respectively $(p=0.001)$ (Table 6$)$. In terms of specific cerebellar dimensions measured from structural MRI scans, there were significant differences in the cerebellar-hemisphere thickness $(p=0.025)$ and right cerebellar-hemisphere longitudinal diameter $(p=0.047)$ between the rs9652490-mutation and non-mutation groups. In contrast, there was no significant difference in the mean ratio of the cerebellar-hemisphere thickness to the fourth-ventricle height between subjects with the rs9652490 mutation $(1.50 \pm 0.42)$ and those without this mutation $(1.70 \pm 0.48)(p=0.060)$. Furthermore, there were no significant differences in the cerebellar-vermis thickness $(p=0.179)$, left cerebellar-hemisphere longitudinal diameter $(p=0.246)$, right or average middle-cerebellar-peduncle width $(p=0.421$ and $p=0.673)$, cerebellar diameter $(p=0.193)$, head diameter $(p=0.877)$, height of the fourth ventricle $(p=0.351)$, or the width of the left middle cerebellar peduncle $(p=0.975)$ between the rs9652490-mutation and non-mutation groups (Table 7).

\section{Discussion}

To the best of our knowledge, this is the first study that investigated correlations of VPA-induced tremor with cerebellar morphology (in structural MRI scans) and SNP rs9652490 within LINGO-1, in which we explored possible predictive factors, along with mechanistic clues of cerebellar dysfunction from clinical practice. Although findings in our cohort did not reveal any association between VPA-induced tremor and SNP rs9652490 within LINGO-1 (an ET risk gene) or cerebellar atrophy, we confirmed a role of the SNP rs9652490 within LINGO-1 in cerebellar atrophy and volumetric loss. Additionally, we verified several clinical factors correlated with VPA-induced tremor in a relatively large cohort.

For the clinical risk factors, the commonly detected risk factors for VPA-induced tremor included VPA therapy duration and daily dosage of VPA, whereas unrelated factors included seizure types, disease duration, abnormalities in neuroimaging/EEGs, the findings of which are similar to those of previous studies[3, 4, 8-10].

A family history of tremor (PD excluded) was an important factor associated with VPA-induced tremor is our cohort, which has rarely been reported in prior studies. Only one previous study observed tremor in one patient with a family history of tremor, at four months after VPA treatment, as compared with that in patients without a family history of tremor with more than 12 months of VPA treatment[4]. This finding suggests an important effect of genetic background in the risk of VPA-induced tremor. In our present study, we found that $20.2 \%$ of subjects in the tremor group had a family history, whereas only $3.5 \%$ of subjects presented with a positive family history in the non-tremor group, indicating that a positive genetic background increases the risk of VPA-induced tremor. Additionally, in our cohort, women were more likely to suffer from VPA-induced tremor, which is consistent with findings from limited studies using scales to measure tremor severity that indicated that women had higher scores in the Clinical Rating Scale for Tremor $(\mathrm{CRST})[9,10]$.

Similar to conclusions of many previous studies, we found that the most prominent risk factors for VPAinduced tremor included VPA usage factors, including treatment duration, daily dosage, and formulations $[3$, $4,8-10]$. In our present study, the VPA therapy duration of the tremor group was longer than that of the non-tremor group ( $\mathrm{p}=0.008)$, and durations of beyond 12 or 24 months increased the incidence of tremor, which is consistent with a recent study demonstrating that the median time from medication to tremor appearance was one year (range of one month to 30 years)[9]. The time from therapy to tremor appearance has been reported to vary from one to 12 months across different cohorts[3, 4, 32], which might be ascribed to small sample sizes and diverse evaluation methods. For example, more sensitive measurements - such as via accelerometers and variable-capacitance transducers - have been shown to enable earlier detection of otherwise undetectable tremors[3, 4]. Prior studies have also indicated that tremors usually occur when the daily VPA dosage is over $750 \mathrm{mg}, 1,000 \mathrm{mg}[3,4]$, or $17.05 \mathrm{mg} / \mathrm{kg}[9]$. In our present study, the incidence of tremor was increased only when the daily dosage exceeded 1,000 mg; we hypothesize that our exclusion of patients taking less than $500 \mathrm{mg} /$ day of VPA might be responsible for our higher cut-off value.

Interestingly, we found that VPA magnesium decreased the risk of tremor, which has never been reported in 
any previous studies. We speculate that a possible mechanism for this phenomenon derives from increased magnesium serum levels, which may induce the following effects: (1) competitive antagonism of the presynaptic binding site of calcium and inhibition of the release of presynaptic vesicles, putatively stabilizing the neural circuits that modulate tremor [33]; (2) lowering of serum calcium concentrations via regulating the secretion of parathormone[34]; (3) elevated magnesium and reduced calcium concentrations may synergistically depress all synaptically transmitted responses, which would further reduce the excitability of the nervous system[33, 34]. Furthermore, the half-life of extended-release VPA magnesium $(13.3-21.3 \mathrm{~h})$ is longer than that of any other formulation of VPA, which consequently confers more stable plasma concentrations and circumvents excessive peak concentrations, which promote lower incidence of side effects of VPA-induced tremor $[8,35]$.

Our present study also revealed a higher risk of tremor in the VPA polytherapy group, with a higher ratio of refractory epilepsy and a longer therapy duration compared to these parameters in the monotherapy group, indicating that VPA interactions with other AEDs may contribute to the incidence of tremor. In a previous study, the number of AEDs was found to not impact the incidence of tremor[7]. However, the severity scores of CRST and Tremor Related Activities of Daily Living (TRADL) were both higher in patients under polytherapy than under monotherapy[9]. Additionally, we found that a combination of VPA with CBZ was found to be beneficial for ameliorating VPA-induced tremor. We consider that the following two points may explain this finding. First, as a potent inducer of hepatic enzymes, CBZ may lower serum concentrations of VPA; in contrast, a combination of VPA and OXC in our cohort did not significantly influence the incidence of tremor, possibly due to its relatively reduced effect on modulating hepatic enzymes. Second, CBZ has been shown to mitigate cerebellar tremor, as evaluated by clinical rating scales and by accelerometric recordings[36]. Although TPM has been reported to be effective for treating moderate to severe ET at a daily dosage of $400 \mathrm{mg}[37,38]$, our present study failed to find a reduced incidence of tremor when VPA was combined with TPM as compared with that of VPA combined with other AEDs, which may have been due to the lower daily dose of TPM in our present study. In addition, we should emphasize that although VPAinduced tremor is similar to ET, the effects of TPM on ET cannot be extrapolated to VPA-induced tremor. From a pharmacokinetic point of view, LTG, which increases plasma levels of VPA, might increase the risk of VPA-induced tremor. In an add-on-therapy trial, LTG significantly increased the incidence of tremor by $4 \%$ compared with that of only $1.8 \%$ for placebo. However, in our present cohort, no difference between groups was found with VPA combined with LTG compared with VPA combined with other AEDs, which might due to the relatively low daily dosage of VPA $(879.31 \pm 241.43 \mathrm{mg} / \mathrm{d})$ or may indicate that variations in metabolism and plasma levels are not primary factors affecting VPA-induced tremor.

Based on the fact that VPA-induced tremor resembles ET[3, 4, 9-11] and that cerebellar dysfunction is hypothesized as a mechanism of ET[20], we explored the associations of VPA-induced tremor with cerebellar atrophy (on MRI scans) and SNP rs9652490 within LINGO-1. However, our data demonstrated that there was no correlation between VPA-induced tremor and these two parameters, which suggests that VPA-induced tremor occurs via a non-LINGO-1 pathway and non-cerebellar locus. Consistent with this finding, VPAinduced tremor rarely presents along with clinical cerebellar symptoms, such as ataxia, gait, or language abnormalities; furthermore, previous conclusive effects of VPA on morphological changes in the cerebellum derive from the fetal developing cerebellum rather than the mature cerebellum[39, 40]. Taken together, the influence of VPA on the volume of the mature cerebellum remains unclear.

Several other pharmacological actions of VPA may provide clues as to its mechanisms in ameliorating tremor. VPA regulates the expression of tyrosine hydroxylase[41], which is the rate-limiting enzyme in the biosynthesis of dopamine, norepinephrine, and epinephrine[42]. Patients taking VPA exhibit lower levels of dopamine and norepinephrine comparing with those of controls[8]. Similarly, reduced dopamine and norepinephrine levels have also been reported in ET patients[8]. Furthermore, VPA induces dopaminergic toxicity and induces injury to the dopaminergic system in experimental mice[43]. Additionally, metabolic dysfunction related to accumulated toxicity has also been reported in patients undergoing VPA treatment[44, 45]. VPA also increases GABAergic neurotransmission in specific brain areas[46, 47]. In one previous study, an increase in the GABA synthesis rate was correlated with tremors and parkinsonism induced by VPA[48]. 
In our present cohort, patients with SNP rs9652490 within LINGO-1 presented with cerebellar atrophy $(p$ $=0.001)$ and reduced values of two dimensions in the cerebellum, namely cerebellar-hemisphere thickness $(p$ $=0.025)$ and right cerebellar-hemisphere longitudinal diameter $(p=0.047)$. Cerebellar hemispheres consist of Purkinje cells and their projection fibers and their morphological atrophy on MRI scans may reflect dysfunction of Purkinje cells. Our present findings support previous findings on the effects of SNP rs9652490 within LINGO-1 on the development and survival of cerebellar Purkinje cells, further corroborating the cerebellar dysfunction hypothesis in ET. This finding also suggests that the mechanisms of VPA-induced tremor may not be limited to the cerebellum. Aside from the cerebellum, tremor may also be generated from dysfunction involved in cortico-olivo-cerebello-thalamic circuits[49].

The current study had several limitations. First, potential recall bias was inevitable. Second, our relatively small sample size may have reduced the association of VPA-induced tremor with SNP rs9652490 within LINGO-1, considering the SNP rs9652490 frequency and racial differences in this mutation. Third, the cerebellar dimensions that we measured may not have been sufficient to accurately reflect volumetric loss in the cerebellum. Hence, voxel-based morphometry should be further used to evaluate cerebellar atrophy, and matched healthy controls should be included as well. With the exception of detecting morphological changes, functional MRI is likely more sensitive in detecting cerebellar dysfunction and related neural-circuit aberrations and may help to better illustrate the underlying mechanisms of VPA-induced tremor in future studies.

In summary, we found that various clinical factors are involved in the risk of VPA-induced tremor. Our cohort confirmed several important and repeatable risk factors, including being female, having a positive family history of tremor (PD excluded), a daily dosage of VPA greater than 1,000 mg/d, a longer treatment duration beyond 12 or 24 months, polytherapy. We also revealed protective factors, including VPA magnesium and a combination of VPA with CBZ. Most interestingly, although VPA-induced tremor and ET manifest similarly, neither of these two factors are associated with ET; similarly, cerebellar atrophy and SNP rs9652490 within LINGO-1 were not correlated with VPA-induced tremor in our present study. Furthermore, our data confirmed that cerebellar atrophy and reductions in two cerebellum-hemisphere dimensions were correlated with SNP rs9652490 within LINGO-1.

\section{Acknowledgments}

The authors would like to thank the patients and their families for their participation, as well as all referring physicians.

\section{Conflicts of interest}

The authors declare no conflicts of interest.

\section{Funding information}

This work was supported by grants from The National Key R\&D Program of China (Grant NO. 2017YFC1310000), National Natural Science Foundation of China (Grant NO. 81974279), China Association Against Epilepsy Fund for Epilepsy Research-UCB fund (Grant NO. 2020020A), the Fundamental Research Funds for the Central Universities (Grant NO. 2018KFYXMPT015), and the Hubei Technological Innovation Special Fund (CN) (Grant NO. 2019ACA132).

\section{Ethical approval and standards}

This study was approved by the Ethical Committee of Tongji Hospital, affiliated with Huazhong University of Science and Technology, and was performed in accordance with the ethical standards established in the 1964 Declaration of Helsinki and its later amendments. Informed consent was obtained prior to the inclusion of each subject.

\section{Consent for publication}

Each patient signed an informed consent for publication of their acquired data. 


\section{Availability of data and materials}

The data that support the findings of this study are available upon request to the corresponding author. The data are not publicly available due to privacy-related and ethical restrictions.

\section{References}

1. Perucca E. Pharmacological and therapeutic properties of valproate: a summary after 35 years of clinical experience. CNS Drugs 2002; 16: 695-714.

2. Davis R, Peters DH, McTavish D. Valproic acid. A reappraisal of its pharmacological properties and clinical efficacy in epilepsy. Drugs 1994; 47: 332-72.

3. Karas BJ, Wilder BJ, Hammond EJ, Bauman AW. Valproate tremors. Neurology 1982; 32: 428-32.

4. Mattson RH, Cramer JA. Tremor due to sodium valproate. Neurology 1981; 31: 114.

5. van der Zwan A, Jr. [Transient Parkinson syndrome and tremor caused by the use of sodium valproate]. Ned Tijdschr Geneeskd 1989; 133: 1230-2.

6. Mehndiratta MM, Satyawani M, Gupta S, Khwaja GA. Clinical and surface EMG characteristics of valproate induced tremors. Electromyogr Clin Neurophysiol 2005; 45: 177-82.

7. Zadikoff C, Munhoz RP, Asante AN, Politzer N, Wennberg R, Carlen P, Lang A. Movement disorders in patients taking anticonvulsants. J Neurol Neurosurg Psychiatry 2007; 78: 147-51.

8. Hamed SA, Abdellah MM. The relationship between valproate induced tremors and circulating neurotransmitters: a preliminary study. Int J Neurosci 2017; 127: 236-42.

9. Xiao Y, Xiong W, Lu L, Chen J, Zhang Y, Jiang X, Zhou D. The clinical characteristics and related factors of tremor in patients with epilepsy. Seizure 2019; 66: 70-75.

10. Alonso-Juarez M, Torres-Russotto D, Crespo-Morfin P, Baizabal-Carvallo JF. The clinical features and functional impact of valproate-induced tremor. Parkinsonism Relat Disord 2017; 44: 147-50.

11. Alonso-Juarez M, Baizabal-Carvallo JF. Distinguishing features between valproate-induced tremor and essential tremor. Acta Neurol Scand 2018; 138: 177-81.

12. Morgan JC, Sethi KD. Drug-induced tremors. Lancet Neurol 2005; 4: 866-76.

13. Hopfner F, Helmich RC. The etiology of essential tremor: Genes versus environment. Parkinsonism Relat Disord 2018; 46 Suppl 1: S92-S96.

14. Bares M, Husarova I, Lungu OV. Essential tremor, the cerebellum, and motor timing: towards integrating them into one complex entity. Tremor Other Hyperkinet Mov (N Y) 2012; 2.

15. Stolze H, Petersen G, Raethjen J, Wenzelburger R, Deuschl G. The gait disorder of advanced essential tremor. Brain 2001; 124: 2278-86.

16. Pagan FL, Butman JA, Dambrosia JM, Hallett M. Evaluation of essential tremor with multi-voxel magnetic resonance spectroscopy. Neurology 2003; 60: 1344-7.

17. Jenkins IH, Bain PG, Colebatch JG, Thompson PD, Findley LJ, Frackowiak RS, Marsden CD, Brooks DJ. A positron emission tomography study of essential tremor: evidence for overactivity of cerebellar connections. Ann Neurol 1993; 34: 82-90.

18. Babij R, Lee M, Cortes E, Vonsattel JP, Faust PL, Louis ED. Purkinje cell axonal anatomy: quantifying morphometric changes in essential tremor versus control brains. Brain 2013; 136: 3051-61.

19. Louis ED, Faust PL, Vonsattel JP, Honig LS, Rajput A, Robinson CA, Rajput A, Pahwa R, Lyons KE, Ross GW, Borden S, Moskowitz CB, Lawton A, Hernandez N. Neuropathological changes in essential tremor: 33 cases compared with 21 controls. Brain 2007; 130: 3297-307. 
20. Stefansson H, Steinberg S, Petursson H, Gustafsson O, Gudjonsdottir IH, Jonsdottir GA, Palsson ST, Jonsson T, Saemundsdottir J, Bjornsdottir G, Bottcher Y, Thorlacius T, Haubenberger D, Zimprich A, Auff E, Hotzy C, Testa CM, Miyatake LA, Rosen AR, Kristleifsson K, Rye D, Asmus F, Schols L, Dichgans M, Jakobsson F, Benedikz J, Thorsteinsdottir U, Gulcher J, Kong A, Stefansson K. Variant in the sequence of the LINGO1 gene confers risk of essential tremor. Nat Genet 2009; 41: 277-9.

21. Kuhlenbaumer G, Hopfner F, Deuschl G. Genetics of essential tremor: meta-analysis and review. Neurology 2014; 82: 1000-7.

22. Clark LN, Park N, Kisselev S, Rios E, Lee JH, Louis ED. Replication of the LINGO1 gene association with essential tremor in a North American population. Eur J Hum Genet 2010; 18: 838-43.

23. Tan EK, Teo YY, Prakash KM, Li R, Lim HQ, Angeles D, Tan LC, Au WL, Yih Y, Zhao Y. LINGO1 variant increases risk of familial essential tremor. Neurology 2009; 73: 1161-2.

24. Wu YW, Rong TY, Li HH, Xiao Q, Fei QZ, Tan EK, Ding JQ, Chen SD. Analysis of Lingo1 variant in sporadic and familial essential tremor among Asians. Acta Neurol Scand 2011; 124: 264-8.

25. Zhou ZD, Sathiyamoorthy S, Tan EK. LINGO-1 and Neurodegeneration: Pathophysiologic Clues for Essential Tremor. Tremor Other Hyperkinet Mov (N Y) 2012; 2.

26. Delay C, Tremblay C, Brochu E, Paris-Robidas S, Emond V, Rajput AH, Rajput A, Calon F. Increased LINGO1 in the cerebellum of essential tremor patients. Mov Disord 2014; 29: 1637-47.

27. Vilarino-Guell C, Ross OA, Wider C, Jasinska-Myga B, Cobb SA, Soto-Ortolaza AI, Kachergus JM, Keeling BH, Dachsel JC, Melrose HL, Behrouz B, Wszolek ZK, Uitti RJ, Aasly JO, Rajput A, Farrer MJ. LINGO1 rs9652490 is associated with essential tremor and Parkinson disease. Parkinsonism Relat Disord 2010; 16: 109-11.

28. Fisher RS, Acevedo C, Arzimanoglou A, Bogacz A, Cross JH, Elger CE, Engel J, Jr., Forsgren L, French JA, Glynn M, Hesdorffer DC, Lee BI, Mathern GW, Moshe SL, Perucca E, Scheffer IE, Tomson T, Watanabe M, Wiebe S. ILAE official report: a practical clinical definition of epilepsy. Epilepsia 2014; 55: 475-82.

29. Haubenberger D, Hallett M. Essential Tremor. N Engl J Med 2018; 378: 1802-10.

30. Elble R, Comella C, Fahn S, Hallett M, Jankovic J, Juncos JL, Lewitt P, Lyons K, Ondo W, Pahwa R, Sethi K, Stover N, Tarsy D, Testa C, Tintner R, Watts R, Zesiewicz T. Reliability of a new scale for essential tremor. Mov Disord 2012; 27: 1567-9.

31. Elble R, Bain P, Forjaz MJ, Haubenberger D, Testa C, Goetz CG, Leentjens AF, Martinez-Martin P, Pavy-Le Traon A, Post B, Sampaio C, Stebbins GT, Weintraub D, Schrag A. Task force report: scales for screening and evaluating tremor: critique and recommendations. Mov Disord 2013; 28: 1793-800.

32. Scheffer IE, Berkovic S, Capovilla G, Connolly MB, French J, Guilhoto L, Hirsch E, Jain S, Mathern GW, Moshe SL, Nordli DR, Perucca E, Tomson T, Wiebe S, Zhang YH, Zuberi SM. ILAE classification of the epilepsies: Position paper of the ILAE Commission for Classification and Terminology. Epilepsia 2017; 58: $512-21$.

33. Long S, Romani AM. Role of Cellular Magnesium in Human Diseases. Austin J Nutr Food Sci 2014; 2.

34. Czeh G, Somjen GG. Changes in extracellular calcium and magnesium and synaptic transmission in isolated mouse spinal cord. Brain Res 1989; 486: 274-85.

35. Rinnerthaler M, Luef G, Mueller J, Seppi K, Wissel J, Trinka E, Bauer G, Poewe W. Computerized tremor analysis of valproate-induced tremor: a comparative study of controlled-release versus conventional valproate. Epilepsia 2005; 46: 320-3.

36. Sechi GP, Zuddas M, Piredda M, Agnetti V, Sau G, Piras ML, Tanca S, Rosati G. Treatment of cerebellar tremors with carbamazepine: a controlled trial with long-term follow-up. Neurology 1989; 39: 1113-5. 
37. Ondo WG, Jankovic J, Connor GS, Pahwa R, Elble R, Stacy MA, Koller WC, Schwarzman L, Wu SC, Hulihan JF, Topiramate Essential Tremor Study I. Topiramate in essential tremor: a double-blind, placebocontrolled trial. Neurology 2006; 66: 672-7.

38. Connor GS. A double-blind placebo-controlled trial of topiramate treatment for essential tremor. Neurology 2002; 59: 132-4.

39. Ingram JL, Peckham SM, Tisdale B, Rodier PM. Prenatal exposure of rats to valproic acid reproduces the cerebellar anomalies associated with autism. Neurotoxicol Teratol 2000; 22: 319-24.

40. Nicolini C, Fahnestock M. The valproic acid-induced rodent model of autism. Exp Neurol 2018; 299: $217-27$.

41. Schreur L, Middeljans-Tijssen CW, Hengstman GJ, Olde Rikkert MG. [Cognitive impairment and parkinsonism due to use of sodium valproate]. Tijdschr Gerontol Geriatr 2009; 40: 29-33.

42. D’Souza A, Onem E, Patel P, La Gamma EF, Nankova BB. Valproic acid regulates catecholaminergic pathways by concentration-dependent threshold effects on TH mRNA synthesis and degradation. Brain Res 2009; 1247: 1-10.

43. Vamos E, Csati A, Vecsei L, Klivenyi P. Effects of valproate on the dopaminergic system in mice. Neurol Res 2009; 31: 217-9.

44. Masmoudi K, Gras-Champel V, Bonnet I, Pannier M, Masson H, Rosa A, Andrejak M. [Dementia and extrapyramidal problems caused by long-term valproic acid]. Therapie 2000; 55: 629-34.

45. Masmoudi K, Gras-Champel V, Masson H, Andrejak M. Parkinsonism and/or cognitive impairment with valproic acid therapy: a report of ten cases. Pharmacopsychiatry 2006; 39: 9-12.

46. Loscher W. Valproate: a reappraisal of its pharmacodynamic properties and mechanisms of action. Prog Neurobiol 1999; 58: 31-59.

47. Loscher W. Effects of the antiepileptic drug valproate on metabolism and function of inhibitory and excitatory amino acids in the brain. Neurochem Res 1993; 18: 485-502.

48. Kanner AM, Balabanov A. Valproate: a practical review of its uses in neurological and psychiatric disorders. Expert Rev Neurother 2002; 2: 151-65.

49. Helmich RC, Toni I, Deuschl G, Bloem BR. The pathophysiology of essential tremor and Parkinson's tremor. Curr Neurol Neurosci Rep 2013; 13: 378.

Figure legend

Figure 1 Schematic diagram of cerebellar dimensions measured via structural MRI. A-C Measurements were made axially at the median plane of the middle cerebellar peduncle. Note that the line from a to $\mathrm{b}$ represents cerebellar-vermis thickness, which was the anteroposterior diameter of vermis; $\mathrm{c}$ to $\mathrm{d}$ and e to $\mathrm{f}$ represent the right and left middle-cerebellar-peduncle widths, respectively, which were the widest diameters of the middle cerebellar peduncles; $g$ to $\mathrm{h}$ represents the cerebellar diameter, which was the widest diameter of the cerebellum; i to $\mathrm{j}$ represents the head diameter, which was the widest diameter from the left to right skull external lamina measured in the same slice as that for the cerebellar diameter. D-F was measured from a sagittal view. The line from $\mathrm{k}$ to $\mathrm{l}$ represents cerebellar-hemisphere thickness, which was the diameter from the back of the vertex of the fourth ventricle to the back edge of the cerebellum at the midsagittal plane; $\mathrm{m}$ to $\mathrm{n}$ represents the cerebellar-hemisphere longitudinal diameter, which was the largest longitudinal diameter measured at the paramedian sagittal plane; o to p represents the fourth-ventricle height, which was the largest diameter from the roof to the bottom of the fourth ventricle at the median sagittal plane. 

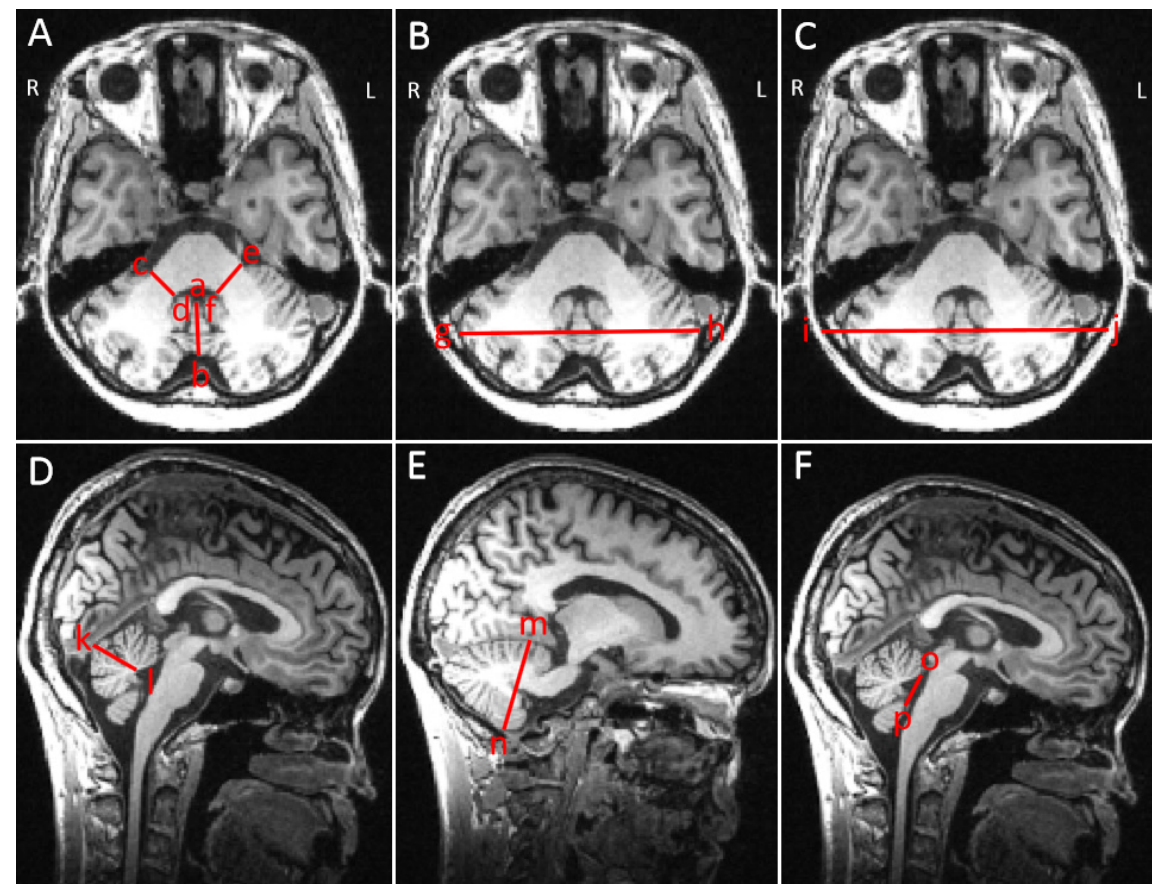

\section{Hosted file}

Tables.docx available at https://authorea.com/users/346911/articles/472717-transversalinvestigation-of-the-risk-of-valproic-acid-induced-tremor-in-a-secondary-epilepsycenter-clinical-neuroimaging-and-genetic-factors 